\title{
Metodología Para La Evaluación De Los Modelos De Gestión De Empendimientos Turisticos: Su Aplicación En El Cantón Puerto Francisco De Orellana - Ecuador
}

\author{
Rita Sulema Lara Vásconez \\ Flor Quinchuela \\ Eduardo Muñoz
}

Docentes investigadores Escuela de Ingeniería en Ecoturismo/

Escuela Superior Politécnica de Chimborazo/Ecuador

doi: 10.19044/esj.2016.v12n11p580 URL:http://dx.doi.org/10.19044/esj.2016.v12n11p580

\begin{abstract}
The present investigation is carried out in the nature of an evaluation of the impact of models of management, taking them as the central point of reference to communal tourism, with emphasis on developing a simple direct methodology, which is permitted within the limits of the information gathered and available, to construct something consistent with the inherent concerns and the impacts on the model of management of communal tourism in the county of Puerto Francisco de Orellana. Furthermore, in addition there is a "ranking" among the businesses based on the studied dimensions and the information about the businesses: Napo Wild life Center, San Warmi, Association Pusarra, and the Community Shuar Atahualpa and the studied dimensions in each case, that is: associativity, appropriateness of actions, structures of the costs of the businesses, and finally, environmental effects in order to know if these contribute to determining if the initiatives of communal tourism determine the level of improvement of the conditions of the life of their founders.
\end{abstract}

Keywords: model of management, communal tourism, methodology, associativity, founders, impact.

\section{Resumen}

El diseño y aplicación de una metodología para evaluar los impactos de los modelos de gestión del turismo en el Cantón Puerto Francisco de Orellana permitió, sistematizar las prácticas en los ámbitos económico social y ambiental. 
El proceso de investigación se llevó a cabo en base al análisis de 4 estudios de caso con tres diferentes modelos de gestión asociativo, comunitario, familiar. Se aplicó la matriz histórica, que básicamente permite recuperar la experiencia vivida por los diferentes grupos de emprendedores turísticos. Se diseñaron criterios de medición para comparar los modelos de gestión, los mismos que van en una escala de medición, desde muy buenas practicas con una valoración de 5 que se la definió como aquella que es tan buena que debería replicarse en otros negocios, hasta muy malas prácticas con una valoración de 1 , que significa que es una práctica que no funciona para ningún negocio, y además produce prejuicios para su entorno. A partir de los datos recogidos se construyó la información para aplicar el proceso metodológico en base a comparaciones de los diferentes modelos discriminando los ámbitos ambiental, económico, social y dimensiones comunitaria, familiar y asociativa.

El modelo de gestión comunitario reporta las mejores prácticas con relación a los ámbitos y dimensiones estudiadas, particularmente se destaca el manejo ambiental y económica basada en la propiedad y uso efectivo de los activos.

Palabras clave: Modelo de gestión, turismo comunitario, turismo familiar, turismo asociativo.

\section{Introducción}

El turismo comunitario en el Ecuador se ha presentado como un modelo de gestión que permite la relación de la comunidad con los visitantes desde una perspectiva intercultural en el desarrollo de viajes organizados con la participación consensuada de sus miembros garantizando el manejo adecuado de los Recursos Naturales. (FEPTCE, 2009), Es así que la palabra modelo es un esquema teórico, de un sistema o de una realidad compleja, que se elabora para facilitar su comprensión y el estudio de su comportamiento. (Villamizar, 2012, p.24), “un modelo de gestión en el que la comunidad local aprovecha el patrimonio natural y/o cultural de la región en la que se asienta para desarrollar y ofrecer un servicio turístico caracterizado por la activa participación comunitaria en la planificación y ejecución de acciones conducentes a potenciar el desarrollo sostenible de la población mediante la reinversión de los beneficios derivados de la actividad turística” (Subsecretaria de Gestión Turística, 2012). Por lo tanto, el diseño metodológico para evaluar los impactos en los modelos de gestión cumple una función muy importante, estableciéndose una relación clara y concisa de cada una de las etapas de la intervención, la descripción de cómo se va a realizar la propuesta de intervención y los pasos a seguir para generar una información que mi proyecto requiere. (García 2009, p. 1), El turismo comunitario es considerado como una actividad económica sostenible y una 
oportunidad para cumplir los Objetivos de Desarrollo del Milenio que busca la prosperidad local la equidad social, el control local, la eficiencia en el uso de los recursos, la rentabilidad, entre otros. Es por esto que mediante la sensibilización de todos los actores que intervienen en la actividad turística comunitaria se busca canalizar el gasto de los visitantes hacia los sectores pobres, promover proyectos pilotos y multiplicarlos (MINTUR, 2007).

El turismo comunitario es considerado como una actividad económica sostenible y una oportunidad para cumplir los Objetivos de Desarrollo del Milenio que busca la prosperidad local la equidad social, el control local, la eficiencia en el uso de los recursos, la rentabilidad, entre otros. Es por esto que mediante la sensibilización de todos los actores que intervienen en la actividad turística comunitaria se busca canalizar el gasto de los visitantes hacia los sectores pobres, promover proyectos pilotos y multiplicarlos (MINTUR, 2007).

Las metodologías para evaluar los modelos de gestión de emprendimientos turísticos persiguen conocer evaluar y entender la incidencia de los cambios inducidos por el turismo es así que , El turismo comunitario es considerado como una actividad económica sostenible y una oportunidad para cumplir los Objetivos de Desarrollo del Milenio que busca la prosperidad local la equidad social, el control local, (MINTUR, 2007), en este sentido se intenta apreciar el ámbito económico cómo el turismo ha sido incorporado en el terreno de la gestión económica comunitaria y si ha podido contribuir a generar recursos que aporten beneficios reales a la comunidad o, de lo contrario, si se ha acentuado su situación de pobreza y dependencia, en la dimensión social se intenta apreciar, analizar y entender los cambios inducidos por las actividades turísticas en las instituciones comunitarias, así como en su dinámica y prácticas sociales, en el contexto ambiental se evaluará si, con ocasión de su incursión en el negocio turístico, la comunidad ha emprendido o consolidado sus iniciativas de autogestión relacionadas con la propiedad, posesión y administración de sus tierras y territorios; el manejo y aprovechamiento sostenible de los recursos naturales. (Maldonado, 2005, .p, 12)

Las metodologías para evaluar modelos de gestión de emprendimientos turísticos son consideraras como una nueva alternativa para generar desarrollo local, la concepción endógena de desarrollo local, supone entender el mismo como un proceso liderado por los actores locales, que tiene la finalidad de mejorar la calidad de vida y satisfacer de manera creciente las necesidades de la población. (Alburquerque, 2004), La puesta en marcha de emprendimientos de turismo comunitario en el Ecuador en general ha contado desde hace varios años con el apoyo de diversas fuentes financieras como gobiernos locales, ONG nacionales e internacionales. En este sentido, el Turismo Comunitario inicia sus actividades en forma paralela 
al turismo convencional a partir de los años 80, que ya para el año 2006 ocupaba a 15000 personas y más de 100 comunidades campesinas e indígenas (Ruiz, 2007, p. 22), Sin embargo, a finales de esa misma década (los 80s) algunas comunidades empezaron a incursionar en la actividad turística desarrollando una nueva propuesta: el turismo comunitario (Torres, 2013, ). Desde que la cooperación para el desarrollo se comprometió en el marco de la « Declaración del Milenio » y la « Declaración de París 2005 » con la observación de objetivos de desarrollo consensuados, la orientación a efectos e impactos está experimentando un alto prestigio a nivel internacional. Más que nunca se le exige a la comunidad de donantes, pero también a las organizaciones no gubernamentales (ONG), que deben dar muestras fehacientes de su capacidad de usar con eficacia los recursos puestos a su disposición, lo que se traduce en un aporte visible al desarrollo de los países contrapartes, (Mogge, 2008).

El Plan Nacional del Buen Vivir, como uno de los ejes transversales más importantes para la consolidación de la nueva matriz productiva del Ecuador plantea objetivos fundamentales tales como: mejorar la calidad de vida de la población, garantizar los derechos de la naturaleza y promover un ambiente sano y saludable, en este contexto el Gobierno Nacional del Ecuador por intermedio de la (SENPLADES, 2013) el Plan Nacional del Buen Vivir 2013-2017, la Constitución de la República del Ecuador 2008, apunta a la consolidación de un nuevo modelo de gestión, convirtiéndose la política pública del Ecuador y los diferentes instrumentos de planificación institucional (planes plurianuales de inversión; planes operativos anuales; agendas sectoriales, planes de desarrollo territorial de los gobiernos autónomos descentralizados), en herramientas de gestión para avanzar en la erradicación de la pobreza , discriminación y garantizar la igualdad de los derechos de todos y todas.

Por la importancia de estudiar de forma comparada las semejanzas y diferencias de los modelos de gestión de turismo comunitario en pro de evaluarlos, la presente investigación contribuye como un aporte científico a la sistematización de buenas prácticas , generación de recursos económicos , mejora de calidad de vida del sector rural, en el Cantón Puerto Francisco de Orellana Provincia de Orellana , que posee recursos naturales y culturales de importancia, cabe recalcar además que los estudios de caso de las operaciones de turismo comunitario se realizaron en poblaciones Quichwa y Shuar de la Amazonia Ecuatoriana, zona de influencia declarada Reserva de Biosfera por la UNESCO y Parque Nacional Yasuni

\section{Método}

Para la presente investigación se aplicaron los métodos y técnicas de investigación de campo y documental. 


\section{Diseño}

Se analizaron 4 estudios de caso con 3 diferentes modelos de gestión: Asociativo, comunitario y familiar. Las iniciativas a estudiar están ubicadas en las comunidades de: Añango, Sani Warmi, Limoncocha y Shuar Atahualpa pertenecientes al Cantón Puerto Francisco de Orellana.

\section{Criterios de medición}

Para construir las matrices de discriminación de iniciativas, se procede a comparar los resultados de la aplicación de las encuestas en función de los siguientes criterios: Encuesta-Anexo1.

Tabla 1. Criterios para estimación de desempeño

\begin{tabular}{|l|l|l|}
\hline Criterios & Definición & Equivalencia \\
\hline $\begin{array}{l}\text { Muy buena } \\
\text { práctica }\end{array}$ & $\begin{array}{l}\text { Se define como aquella que es tan buena que debería replicarse } \\
\text { a otros negocios. }\end{array}$ & 5 \\
\hline $\begin{array}{l}\text { Buena } \\
\text { práctica }\end{array}$ & $\begin{array}{l}\text { Definida como aquella práctica que funciona al momento del } \\
\text { análisis para el negocio que la obtuvo. }\end{array}$ & 4 \\
\hline $\begin{array}{l}\text { Indiferente } \\
\text { o no aplica }\end{array}$ & $\begin{array}{l}\text { Que puede además implicar que no existen datos suficientes } \\
\text { para poder evaluarla, o que al momento de la encuesta no se } \\
\text { pudo obtener, o el informante no lo proporcionó. }\end{array}$ & 3 \\
\hline $\begin{array}{l}\text { Mala } \\
\text { práctica }\end{array}$ & $\begin{array}{l}\text { Es una práctica de negocios que no funciona para ese modelo } \\
\text { de negocios. }\end{array}$ & 2 \\
\hline $\begin{array}{l}\text { Muy mala } \\
\text { práctica }\end{array}$ & $\begin{array}{l}\text { Es una práctica que no funciona para ningún negocio y además } \\
\text { produce perjuicios para su entorno. }\end{array}$ & 1 \\
\hline
\end{tabular}

Inmediatamente, en función de las encuestas realizadas a los negocios se trabajó la valoración de los criterios para los negocios y posteriormente se tabularon los resultados para construir las matrices respectivas.

\section{Evaluación a los diferentes modelos de gestión del turismo comunitario.}

Se aplicaron las herramientas ya diseñadas en los ámbitos correspondientes, en los emprendimientos estudiados en las comunidades y los grupos organizados que se visitaron.

\section{Resultados y discusión Napo Wildlife Center}

Este negocio surge en 1998, época en la que 10 habitantes de la comunidad Añangu, todos ex trabajadores de la empresa Sacha Lodge En los primeros meses de actividad ofrecían únicamente servicios de camping y ya tuvieron visitantes extranjeros, uno de ellos se interesó por el negocio, y a través de la empresa Tropical Nature posibilitó el apoyo técnico y financiero por un millón de dólares Entre las principales motivaciones para arrancar y mantener el negocio desde la población local, figura la necesidad de crear y 
generar empleo rural no agropecuario, que evite la migración y sus secuelas sociales.

\section{Sani Warmi}

A raíz del éxito del emprendimiento de la comunidad vecina Añangu, la comunidad Sani empieza también a mirar al turismo como una opción. Al momento se ha logrado el apoyo de la empresa petrolera Occidental que finalmente construyó y capacitó en servicios hoteleros a los habitantes de la comunidad. Desde el arranque de las operaciones se ofreció transporte, alimentación, hospedaje, transporte, guía. Se abrió también una oficina en la ciudad de Quito.

\section{Asociación Pusara - Limoncocha}

Este emprendimiento tuvo su origen en una reunión comunitaria que contó como protagonista principal a uno de sus habitantes, el Sr. Germán Tanguila, quien había trabajado previamente como empleado de la empresa Sacha Lodge.

El escenario principal de este negocio es la reserva biológica Limoncocha, inicialmente operaron con 50 socios y socias, desde el año 2010, según sus directivos además de la necesidad de generar empleo local su emprendimiento es una respuesta a las necesidades del mercado, que reclamaban en ese momento la mejora de las condiciones para el hospedaje de los mochileros, de camping a cabañas.

Esta organización no recibió apoyo en su etapa inicial, los gastos de arranque, estimados en 5000 USD y fueron producto de las aportaciones de los socios. Los servicios que ofrecían eran alimentación hospedaje y guía.

\section{Comunidad Shuar - Atahualpa}

El impulso inicial de este emprendimiento vino de un comunero llamado Luis Yankuam, quien conocía los potenciales beneficios del turismo para la comunidad, luego de varias reuniones con los habitantes de la comunidad, aproximadamente 62 de ellos decidieron apoyarle para iniciar la gestión de la idea ante las organizaciones del estado, producto de ello, en el año 2012, comenzaron a buscar el apoyo institucional y fue la delegación provincial de Orellana del Ministerio de Turismo que comenzó con el apoyo para la promoción de la danza en eventos del cantón. Una de las principales motivaciones de esta organización para el emprendimiento, fue la experiencia conocida en otras comunidades cercanas, en la puesta en marcha de iniciativas de turismo comunitario que aprovechaban los escenarios y las potencialidades naturales de sus territorios. Los servicios ofrecidos desde su inauguración son la alimentación y la guía dentro de su zona de influencia. Al momento se está trabajando con el apoyo voluntario de técnicos del 
MINTUR de Orellana, Ministerio de Cultura, estudiantes y docentes de la ESPOCH-ENA.

\section{Asociatividad}

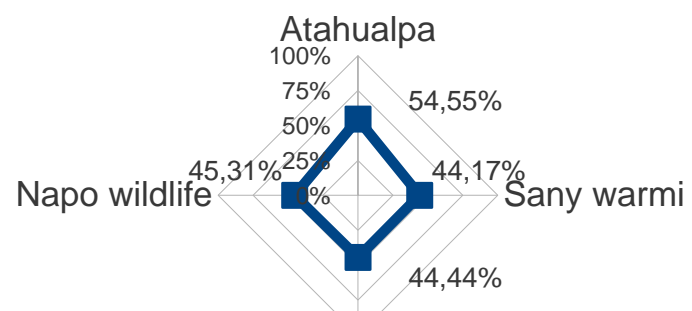

\section{Pusara}

Figura 1: Género y asociatividad Realizado por: Rita Sulema Lara Vásconez

La conformación por género de las sociedades mantiene un ligero sesgo hacia los hombres.

Los porcentajes presentados presentan cierta tendencia hacia la equidad de género y la conformación societaria. Sin embargo en este apartado destaca la organización Atahualpa, con el 54,55 \% de mujeres parte de su organización

Mientras por edades, la conformación societaria, de todos los negocios estudiados se concentran en los rangos entre los 30 y los 49 años, de lo que se observa, la conformación societaria tendrá un nivel mixto de renovación en el mediano plazo.

Tabla 2. Formación académica de los socios y socias de los negocios.

\begin{tabular}{|l|l|l|l|l|l|l|l|l|}
\hline \multirow{2}{*}{$\begin{array}{l}\text { Formación } \\
\text { académica }\end{array}$} & Napo Wildlife & \multicolumn{2}{l|}{ Sany Warmi } & Pusara & \multicolumn{2}{l|}{ Atahualpa } \\
\cline { 2 - 10 } & Hombres & mujeres & hombres & mujeres & hombres & Mujeres & hombres & mujeres \\
\hline No tienen estudios & 3 & 3 & & & & & & \\
\hline Primaria & 32 & 26 & 400 & 200 & 10 & 10 & 14 & 8 \\
\hline Secundaria & & & & & 6 & 3 & & \\
\hline Universidad & & & & & 4 & 3 & & \\
\hline 4to nivel & & & & & & & & \\
\hline Total & 35 & 29 & 400 & 200 & 20 & 16 & 14 & 8 \\
\hline$\%$ & $70 \%$ & $30 \%$ & $67 \%$ & $33 \%$ & $56 \%$ & $44 \%$ & $70 \%$ & $36 \%$ \\
\hline
\end{tabular}

Realizado por: Rita Sulema Lara Vásconez

En cuanto la conformación el nivel de educación formal de los socios y socias el $96 \%$ tiene primaria, el 1\% no tiene estudios formales, el $1 \%$ tiene estudios secundarios y de igual forma el 1\% tiene estudios universitarios. 
Tabla 3. Participación extrasocietaria.

\begin{tabular}{|l|l|l|l|l|l|l|l|l|}
\hline & \multicolumn{2}{|l|}{ Napo Wildife } & \multicolumn{2}{l|}{ Sany Warmi } & \multicolumn{2}{l|}{ Pusara } & \multicolumn{2}{l|}{ Atahualpa } \\
\cline { 2 - 10 } $\begin{array}{l}\text { Participación } \\
\text { extrasocietaria }\end{array}$ & $\begin{array}{l}\text { Hombr } \\
\text { es }\end{array}$ & $\begin{array}{l}\text { mujer } \\
\text { es }\end{array}$ & $\begin{array}{l}\text { hombr } \\
\text { es }\end{array}$ & $\begin{array}{l}\text { Mujer } \\
\text { es }\end{array}$ & $\begin{array}{l}\text { hombr } \\
\text { es }\end{array}$ & $\begin{array}{l}\text { Mujer } \\
\text { es }\end{array}$ & $\begin{array}{l}\text { Hombr } \\
\text { es }\end{array}$ & $\begin{array}{l}\text { mujer } \\
\text { es }\end{array}$ \\
\hline Comunidad & & & & & & & 10 & 12 \\
\hline $\begin{array}{l}\text { Educación de los } \\
\text { hijos/as }\end{array}$ & 3 & 3 & & & 7 & 6 & & \\
\hline Agua de consumo & & & & & 7 & 5 & & \\
\hline Otros negocios & & & & & & & & \\
\hline Otras organizaciones & & & 335 & 265 & 6 & 5 & & \\
\hline Ninguna & 32 & 26 & & & & & & \\
\hline total & 35 & 29 & 335 & 265 & 20 & 16 & 10 & 12 \\
\hline$\%$ & $50 \%$ & $50 \%$ & $50 \%$ & $50 \%$ & $56 \%$ & $44 \%$ & $40 \%$ & $60 \%$ \\
\hline
\end{tabular}

Realizado por: Rita Sulema Lara Vásconez

Las organizaciones Sany Warmi por el número de socios y Pusara por la diversidad de organizaciones destacan en cuanto sus socios y socias son miembros de otras organizaciones comunitarias.

Tabla 4. Interés de socios y socias en las actividades de los negocios.

\begin{tabular}{|l|l|l|l|l|l|l|l|l|}
\hline $\begin{array}{l}\text { Interés de } \\
\text { socios y socias } \\
\text { por actividades } \\
\text { de los negocios }\end{array}$ & hombres & mujeres & Hombres & mujeres & Hombres & Mujeres & Hombres & mujeres \\
\hline Excelente & 35 & 29 & & & & & & \\
\hline Muy Buena & & & 335 & 265 & & & 10 & 12 \\
\hline Buena & & & & & 20 & 16 & & \\
\hline Indiferente & & & & & & & & \\
\hline Mala & & & & & & & & \\
\hline Muy mala & & & & & & & & \\
\hline total & 35 & 29 & 335 & 265 & 20 & 16 & 10 & 12 \\
\hline$\%$ & $55 \%$ & $45 \%$ & $50 \%$ & $50 \%$ & $56 \%$ & $44 \%$ & $45 \%$ & $55 \%$ \\
\hline
\end{tabular}

Fuente: Elaboración propia

Realizado por: Rita Sulema Lara Vásconez

En la totalidad de los emprendimientos la relación de sus socios con su negocio se califica como de buena para arriba, esto es especialmente notorio en Napo Wildlife y en Sani Warmi:

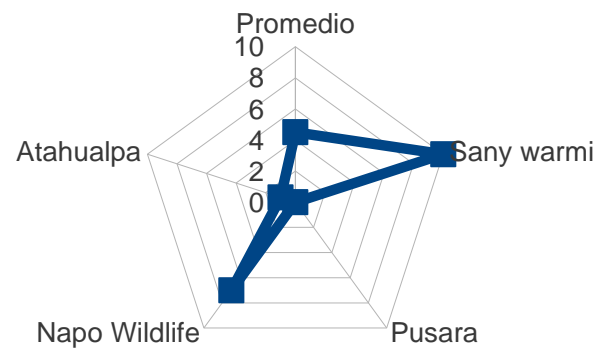

Figura 2: Género y asociatividad 
Desde los socios y socias el conocimiento del manejo del negocio como tal, no es precisamente exacto, pues solamente en las iniciativas Napo Wildlife, y Sany Warmi hay cierto conocimiento sobre el tema financiero, en los otros casos, el conocimiento es inexacto o inexistente, esta situación coincide con el tamaño de los negocios. Mientras, el desarrollo de los productos turísticos en el mercado, el conocimiento mismo del sector, La participación de los socios y socias en la provisión de los servicios que prestan las iniciativas de turismo comunitario es total en las iniciativas Napo Wildlife, Sany Warmi y Atahualpa, solamente en Pusara esta participación aún necesita un mejoramiento importante.

A continuación se presenta un resumen de los resultados. Se utilizó una escala que va desde las muy malas prácticas, hasta las prácticas muy buenas, para ello se valoraron los resultados de las encuestas realizadas durante la preparación de este estudio:

Tabla 5: Matriz de comparación entre negocios - asociatividad

\begin{tabular}{|l|l|l|l|l|}
\hline Asociatividad & $\begin{array}{l}\text { Napo } \\
\text { Wildife } \\
\text { Center }\end{array}$ & $\begin{array}{l}\text { Sany } \\
\text { Warmi }\end{array}$ & $\begin{array}{l}\text { Asociación Pusara } \\
\text { Limoncocha }\end{array}$ & $\begin{array}{l}\text { Comunidad Shuar - } \\
\text { Atahualpa }\end{array}$ \\
\hline Género & 4 & 4 & 4 & 5 \\
\hline Edad otras & 2 & 2 & 5 & 4 \\
\hline Educación por el & 2 & 4 & 5 & 4 \\
\hline $\begin{array}{l}\text { Relación } \\
\text { asociaciones }\end{array}$ & 5 & 5 & 5 & 2 \\
\hline $\begin{array}{l}\text { Interés de socios por } \\
\text { negocio }\end{array}$ & 4 & 4 & 4 \\
\hline Ingreso de nuevos socios/as & 2 & 5 & 2 & 2 \\
\hline $\begin{array}{l}\text { Conocimiento de los socios/as } \\
\text { sobre el negocio }\end{array}$ & 5 & 5 & 2 & 2 \\
\hline $\begin{array}{l}\text { Provisión de servicios desde } \\
\text { los socios/as }\end{array}$ & 5 & 5 & 2 & 5 \\
\hline $\begin{array}{l}\text { Procesos administrados por los } \\
\text { socios/as }\end{array}$ & 4 & 5 & 2 & 2 \\
\hline Comercialización directa & 5 & 4 & 4 & 4 \\
\hline
\end{tabular}

\begin{tabular}{|l|l|l|l|l|}
\hline Muy buena práctica & $40 \%$ & $50 \%$ & $30 \%$ & $20 \%$ \\
\hline Buena práctica & $30 \%$ & $40 \%$ & $30 \%$ & $40 \%$ \\
\hline Indiferente o no aplica & $0 \%$ & $0 \%$ & $0 \%$ & $0 \%$ \\
\hline Mala práctica & $30 \%$ & $10 \%$ & $40 \%$ & $40 \%$ \\
\hline Muy mala práctica & $0 \%$ & $0 \%$ & $0 \%$ & $0 \%$ \\
\hline
\end{tabular}

Realizado por: Rita Sulema Lara Vásconez

De lo que se observa en este apartado Sany Warmi tiene las mejores prácticas en cuanto a asociatividad se refiere.

\section{Propiedad de los activos.}

En el $100 \%$ de los casos los socios y socias son propietarios de las iniciativas de turismo comunitario 
El nivel de activos exigibles en las empresas va en un rango desde las cuatro cifras bajas, hasta las 7 cifras bajas. En iguales niveles se ubicaron las ventas del año 2014.

Nuevamente, en el caso de Napo Wildlife, es la única iniciativa que vende servicios de turismo a crédito, el tamaño y el modelo de gestión favorecen esta práctica comercial

En el Ecuador, las empresas de turismo comunitario han seguido estrategias mixtas de comercialización privilegiando una combinación de comercialización directa y en relación con empresas especializadas en turismo receptivo.

Las empresas operadoras de turismo que tienen ciertas relaciones comerciales con los negocios estudiados en general, y con Pusara en particular son: Agancagua Tours, Cortus, Galeno Lodge, Limoncocha Tours, Mundo Puma, Napo River Amazon, Panky Tours, Sumak Alpa, Witoto Tours. Las otras empresas aun teniendo contactos comerciales no revelan esta información pues son muy celosos del cuidado de sus contactos comerciales.

Un aspecto a resaltar, es que en todos los casos estudiados, no existen práctica ni operativamente activos improductivos, todas las instalaciones, equipos, rutas, entre otros, se están usando para la prestación de los servicios turísticos.

El 75\% de los emprendimientos posee cabañas como forma de hospedaje, en conjunto poseen 31 cabañas, con una capacidad total de 97 pax. La iniciativa con mayor número de cabañas es Napo Wildlife con 16, le sigue Sany Warmi con 14, Pusara con 1 y Atahualpa aún no brinda este servicio.

A continuación se presenta la matriz comparativa entre los negocios estudiados en relación con la propiedad de los activos y el nivel de ventas.

Tabla 6: Matriz de comparación entre negocios - propiedad de los activos y ventas

\begin{tabular}{|l|l|l|l|l|}
\hline Propiedad de los activos & $\begin{array}{l}\text { Napo Wildlife } \\
\text { Center }\end{array}$ & $\begin{array}{l}\text { Sany } \\
\text { Warmi }\end{array}$ & $\begin{array}{l}\text { Asociación Pusara } \\
\text { Limoncocha }\end{array}$ & $\begin{array}{l}\text { Comunidad Shuar - } \\
\text { Atahualpa }\end{array}$ \\
\hline $\begin{array}{l}\text { Propiedad de la } \\
\text { asociación/comunidad }\end{array}$ & 5 & 5 & 5 & 5 \\
\hline Nivel de activos exigibles & 5 & 4 & 4 & 4 \\
\hline Volumen de ventas & 5 & 4 & 4 & 4 \\
\hline Ventas a crédito & 5 & 2 & 2 & 2 \\
\hline Contactos comerciales & 5 & 5 & 5 & 5 \\
\hline Activos improductivos & 5 & 5 & 5 & 5 \\
\hline
\end{tabular}

\begin{tabular}{|l|l|l|l|l|}
\hline Muy buena práctica & $100 \%$ & $50 \%$ & $50 \%$ & $50 \%$ \\
\hline Buena práctica & $0 \%$ & $33 \%$ & $33 \%$ & $33 \%$ \\
\hline Indiferente o no aplica & $0 \%$ & $0 \%$ & $0 \%$ & $0 \%$ \\
\hline Mala práctica & $0 \%$ & $17 \%$ & $17 \%$ & $17 \%$ \\
\hline Muy mala práctica & $0 \%$ & $0 \%$ & $0 \%$ & $0 \%$ \\
\hline
\end{tabular}

Realizado por: Rita Sulema Lara 
En cuanto la propiedad de los activos y las prácticas comerciales sin duda la empresa que lidera este apartado es Napo Wildlife.

\section{Estructura de costos de los negocios}

En cuanto los costos fijos anuales, se logró obtener la información de las empresas Napo Wildlife, Sani Warmi y Pusara, a continuación la comparación absoluta de los valores. Atahualpa no entrego la información requerida: Napo Wildlife con un total de 252516, Sani Lodge 4500, Pusara 108942, el valor más alto lo tiene Napo wild life. Este resultado debido al tamaño del modelo de gestión. Y al tiempo de su puesta en marcha en el mercado desde 1998.

Mientras el volumen de los costos variables se puede apreciar como sigue: Napo Wild Life 11.860, y Pusara 230 en consecuencia de lo anterior, también en este caso el valor más alto de los activos fijos (depreciados) corresponde a Napo Wildlife por el tamaño del modelo de gestión

En cuanto a los activos depreciables encontramos los siguientes valores: Napo Wildlife 252516, Sani Lodge 4500, Pusara 10894.

A continuación se presenta un resumen y la valoración de la estructura de costos de los negocios estudiados

En cuanto a propiedad de los activos la mejor combinación se presenta en Pusara Limoncocha.

\section{Medidas de Mitigación para enfrentar las potenciales afectaciones ambientales}

Afectación del aire en la zona de intervención:

La contaminación producida por los generadores es potencial. Aunque se trata de reducir la emisión de gases tóxicos con la puesta en marcha de medidas de generación eléctrica, paneles solares y baterías, como en el caso de Napo Wildlife.

\section{Afectación del agua en los procesos de provisión de los servicios.}

En los casos estudiados, la afectación a los cauces naturales de las aguas de los emplazamientos de turismo comunitario es mínimo o inexistente. El manejo de desperdicios es más prolijo en Napo Wildlife y Pusara, donde se los clasifica en orgánicos e inorgánicos y posteriormente lo que corresponde se lo vende a los negocios locales de recicladores. El manejo de productos especialmente peligrosos se realiza en áreas especiales de tal forma que se minimice el riesgo de un eventual derrame y en todo caso a estos envases se les da un tratamiento definitivo es decir se los incinera. Para el manejo y mantenimiento de las posibles afectaciones producto de la operación y/o mantenimiento de equipos/maquinaria y vehículos, como: los aceites, el diésel, la gasolina, cuando se derraman sobre el agua si causan un 
daño en la superficie de la misma, al menos en Pusara existe el principio de limpieza inmediata que consiste en : primero delimitar el sitio con barreras especiales de polietileno, plástico , caucho, y utilizar esponjas especiales absorbentes para derrames de combustibles. En general en todas las iniciativas los lugareños afirman que no han disminuido los caudales de agua para el uso de sus comunidades después de iniciada la explotación turística. Mientras, en esta misma línea, Napo Wildlife contempla en su actividad regular el tratamiento de las aguas grises con un mecanismo micro localizados de filtración, y las aguas negras van a un pozo séptico, mientras en Pusara se trabaja con el pozo triaca eral para el tratamiento de las aguas negras En el mismo contexto, En las otras organizaciones la aguas negras se destinan a pozos sépticos., y las aguas grises son expulsadas directamente a los ríos, sin tomar medidas de mitigación al respecto

Afectación del recurso suelo en los procesos de provisión del servicio.

Las explotaciones turísticas comunitarias estudiadas no demandan la tala recurrente en áreas circundantes a senderos u otras facilidades turísticas, mientras el uso de químicos peligrosos como en el caso del cloro compra la cantidad justa, se almacenan en sitios especiales y ante un eventual derrame existe la disposición para realizar la limpieza con exactitud y celeridad. Para el manejo y mantenimiento de las posibles afectaciones producto de la operación y/o mantenimiento de equipos/maquinaria $\mathrm{y}$ vehículos, como: los aceites, el diésel, la gasolina, cuando se derraman sobre el suelo si causan daños, debido a que si no se hace una limpieza correcta del sitio estos combustibles se expanden, causando daños en la vegetación. Al menos en Pusara el principio de limpieza inmediata que consiste en realizar una excavación manual del área afectada por el derrame, ese suelo se lo bota en un lugar destinado para el tratamiento de suelo protegido contra la humedad y la lluvia, bajo una mezcla con aserrín o compost y cepas necesarias para el proceso de oxigenación para la bioremediación.

Tabla 7: Matriz comparativa: Potenciales afectaciones ambientales

\begin{tabular}{|c|l|l|l|l|}
\hline $\begin{array}{l}\text { Potenciales afectaciones } \\
\text { ambientales }\end{array}$ & $\begin{array}{l}\text { Napo Wildlife } \\
\text { Center }\end{array}$ & $\begin{array}{l}\text { Sany } \\
\text { Warmi }\end{array}$ & $\begin{array}{l}\text { Asociación Pusara } \\
\text { Limoncocha }\end{array}$ & $\begin{array}{l}\text { Comunidad Shuar - } \\
\text { Atahualpa }\end{array}$ \\
\hline Aire & & & & \\
\hline Emisión de polvo & 5 & 5 & 5 & 5 \\
\hline Emisión de humo & 4 & 4 & 3 & 3 \\
\hline Emisión de gases & 5 & 3 & 5 & 3 \\
\hline Agua & & & & \\
\hline $\begin{array}{c}\text { Modificación de cauces } \\
\text { naturales de agua }\end{array}$ & 5 & 4 & 5 & 4 \\
\hline Manejo de desperdicios & 5 & 2 & 5 & 2 \\
\hline $\begin{array}{c}\text { Manejo de productos } \\
\text { peligrosos }\end{array}$ & 5 & 5 & 5 & 5 \\
\hline
\end{tabular}




\begin{tabular}{|c|c|c|c|c|}
\hline $\begin{array}{l}\text { Buen uso del agua - } \\
\text { turismo }\end{array}$ & 4 & 4 & 5 & 4 \\
\hline Disminución de caudales & 5 & 5 & 5 & 5 \\
\hline $\begin{array}{lll}\begin{array}{l}\text { Tratamiento } \\
\text { grises }\end{array} & \text { de } & \text { aguas } \\
\end{array}$ & 5 & 1 & 5 & 1 \\
\hline \multicolumn{5}{|l|}{ Suelo } \\
\hline Tala recurrente & 5 & 5 & 5 & 5 \\
\hline $\begin{array}{l}\begin{array}{c}\text { Derrame de sustancias } \\
\text { tóxicas }\end{array} \\
\end{array}$ & 5 & 5 & 5 & 5 \\
\hline Muy buena práctica & $82 \%$ & $45 \%$ & $91 \%$ & $45 \%$ \\
\hline Buena práctica & $18 \%$ & $27 \%$ & $0 \%$ & $18 \%$ \\
\hline Indiferente o no aplica & $0 \%$ & $9 \%$ & $9 \%$ & $18 \%$ \\
\hline Mala práctica & $0 \%$ & $9 \%$ & $0 \%$ & $9 \%$ \\
\hline Muy mala práctica & $0 \%$ & $9 \%$ & $0 \%$ & $9 \%$ \\
\hline
\end{tabular}

Tabla 8: Resumen de resultados - modelo de gestión

\begin{tabular}{|l|l|r|}
\hline Modelo de gestión & \multicolumn{1}{|c|}{ Negocio } & Calificación promedio \\
\hline Comunitario & Napo Wildlife Center & $84.17 \%$ \\
\hline Asociativ o & Asociación Pusara Limoncocha & $83.56 \%$ \\
\hline Asociativo & Sani Warmi & $69.85 \%$ \\
\hline Familiar & Comunidad Shuar - Atahualpa & $60.08 \%$ \\
\hline
\end{tabular}

Tabla9: Resumen de resultados - dimensión estudiada

\begin{tabular}{|l|r|r|r|r|}
\hline $\begin{array}{l}\text { Dimensión/org } \\
\text { anización }\end{array}$ & $\begin{array}{c}\text { Napo } \\
\text { Wildlife } \\
\text { Center }\end{array}$ & Sani Warmi & $\begin{array}{c}\text { Asociación } \\
\text { Pusara } \\
\text { Limoncocha }\end{array}$ & $\begin{array}{c}\text { Comunidad } \\
\text { Shuar - } \\
\text { Atahualpa }\end{array}$ \\
\hline Asociatividad & $70.00 \%$ & $90.00 \%$ & $60.00 \%$ & $60.00 \%$ \\
\hline $\begin{array}{l}\text { Propiedad de } \\
\text { los activos }\end{array}$ & $100.00 \%$ & $83.33 \%$ & $83.33 \%$ & $83.33 \%$ \\
\hline $\begin{array}{l}\text { Estructura de } \\
\text { costos }\end{array}$ & $66.67 \%$ & $33.33 \%$ & $100.00 \%$ & $33.33 \%$ \\
\hline $\begin{array}{l}\text { Potenciales } \\
\text { afectaciones } \\
\text { ambientales }\end{array}$ & $100.00 \%$ & $72.73 \%$ & $90.91 \%$ & $63.64 \%$ \\
\hline Promedio & $84.17 \%$ & $69.85 \%$ & $83.56 \%$ & $60.08 \%$ \\
\hline
\end{tabular}

\section{Conclusion}

En los casos estudiados existe una relación directa entre tamaño del negocio, el grado de información y conocimiento de los socios de su manejo.

El diseño de los criterios de medición para la conformación de las tablas de discriminación permite obtener una visión estandarizada del desempeño de los diferentes modelos de gestión estudiados para los casos analizados, esta metodología simple pero potente permite realizar nuevos acercamientos a esta problemática que se presenta en los negocios turísticos.

Analizados los modelos aplicando la metodología diseñada, el comunitario es aquel que reporta mejores resultados en cuanto al ámbito ambiental y económico basado en la propiedad y uso efectivo de los activos. 
Los niveles de activos tienen una relación directamente proporcional al tamaño de la operación de turismo comunitario. En todos los casos los modelos de gestión aprovechan los activos de los negocios en un $100 \%$, no tienen prácticamente activos improductivos, todas las adquisiciones y desarrollo de productos previos se ocupan regularmente.

Mientras Las medidas de mitigación para enfrentar las potenciales afectaciones ambientales que se ponen en práctica en las explotaciones de turismo comunitario responden las necesidades de conservación y protección, pero además están adaptadas a las posibilidades reales desde los volúmenes de negocio de los negocios comunitarios.

\section{References:}

MALDONADO, 2005. Turismo comunitario reflexiones. Consultado el 31 de mayo del 2015.2 Recuperado de: http://biblio.universidadecotec.edu.ec/revista/edicionespecial/TURISMO\%2 OCOMUNITARIO.pdf

MANN. Mark. (2014). the Community Tourism Guide: Exciting Holidays for Responsible Travellers. Routledge, New York.

MINTUR, 2007. Diseño del Plan Estratégico de Desarrollo de Turismo Sostenible para el Ecuador Plandetur 2020. Mintur Ed. Bid. Quito-Ecuador. MORALES, Javier. Y KREKELER, Jorge. (2012) Autocrítica y diálogo: la orientación a efectos en los proyectos de la cooperación internacional. Misereor, Aachen.

OMT, (2006). La enciclopedia libre. Turismo sostenible. Consultado el 15 de Enero 2014.2 Recuperado de: http://es.wikipedia.org/wiki/Turismo_sostenible. PLANDETUR. (2020). Diseño del Plan Estratégico de Desarrollo de Turismo Sostenible para Ecuador. Recuperado el 15 de Marzo de 2015, de https://www.turismo.gob.ec/PLANDETUR-2020.pdf

VILLAMAR, 2012. Modelo de Gestión. Consultado el 31 de mayo del 2015. Disponible en: http://es.slideshare.net/zulay1719/modelo-de-gestion 